\title{
Klaus Ley (ed): Adhesion molecules: function and inhibition (Series: PIR-Progress in Inflammation Research)
}

\author{
2007, 328 p., Hardcover, Birkhäuser Verlag AG, Basel, Switzerland, \\ ISBN 978-3-7643-7974-2
}

\author{
Andrew Devitt
}

Published online: 24 December 2009

(c) Birkhäuser Verlag, Basel/Switzerland 2009

Whilst leukocyte extravasation across high endothelial venules was first observed in the 1960s our understanding of the molecular mechanisms of this process are much more recent and followed the advent of monoclonal antibody technology. Over the last 25 years our knowledge of adhesion events between leukocytes and endothelia has expanded greatly and continues to expand since the first identification of L-selectin. This knowledge often appears confusing to those outside the field with molecular names being derived perhaps from the alphanumeric code of a crucial monoclonal antibody, a description of the molecule (from its function or apparent pattern of expression), a systematic 'cluster of differentiation' code or all the three. Once past the names, one must get to grips with the detail of the multi-step adhesion process involved in recruiting cells from the blood and into inflamed tissues. This book is a great and informative text to all those who wish to access and demystify the field of adhesion molecules.

The monograph comprises 309 pages in 13 chapters and deals specifically with adhesion molecules in the whole process of leukocyte extravasation with a focus on inflammation. Consequently this book does not detail, though in parts touches upon, the importance of adhesion molecules in a variety of other processes (e.g. phagocytosis). However, with the given focus it provides an extremely competent review of current knowledge (from the historical to the leading edge of adhesion research) of the most important leukocyte adhesion molecules involved in recruitment of inflammatory cells. In doing so it condenses very large amounts of research but crucially also highlights gaps and limitations in our current experimental systems and understanding.

As one might expect from the typically excellent Progress in Inflammation Research series this is a wellorganised and highly informative text. It deals with the multistep recruitment of inflammatory cells from blood to inflamed tissue in three sections (Capture \& Rolling; Firm Adhesion and Transendothelial migration). In each of these sections, individual chapters from major researchers in the field detail the most important molecules. The selectins (L, $\mathrm{P}$ and E), PSGL-1, VAP-1, integrins $\left[\alpha_{4}, \alpha_{6}, \beta_{2}\right.$ (LFA-1 and Mac-1)] and immunoglobulin super-family members (ICAM-1, VCAM-1, PECAM-1, ESAM, JAM members) are all covered with details of molecular structure, expression, ligands/receptors, ligand-binding, signalling and roles in both physiology and pathology.

For the uninitiated, each chapter stands alone and leads the reader usefully through the historical background to the current understanding of given molecules and processes. For the aficionado, it provides food for thought relevant to the application of current knowledge. The final chapter especially provides a balanced view of the potential for modulating adhesion molecules for therapeutic benefit. For something so potentially complicated this is an eminently accessible and readable volume.

\footnotetext{
A. Devitt $(\square)$

Life and Health Sciences, Aston University, Aston Triangle, Birmingham B4 7ET, UK

e-mail: a.devitt1@aston.ac.uk
} 